\title{
Some Properties of Bipolar Fuzzy Cosets of a Bipolar Fuzzy and Bipolar Anti Fuzzy HX Subgroup
}

\author{
R. Muthuraj ${ }^{1}$, M.Sridharan ${ }^{2}$ \\ ${ }^{1}$ PG \& Research Department of Mathematics, H.H.The Rajah's College,Pudukkottai-622 001, Tamilnadu, \\ India. \\ ${ }^{2}$ Department of Mathematics, PSNA College of Engineering and Technology, Dindigul-624 622, Tamilnadu, \\ India.
}

\begin{abstract}
In this paper, we define the concept of a bipolar fuzzy cosets of a bipolar fuzzy HX subgroup, bipolar anti fuzzy HX subgroup, bipolar fuzzy middle coset of a bipolar fuzzy HX subgroup and bipolar anti fuzzy $H X$ subgroup, conjugate bipolar fuzzy $H X$ subgroup, conjugate bipolar anti fuzzy $H X$ subgroup and discussed some of its properties with the examples. Further we define the level subsets and lower level subsets of bipolar fuzzy cosets of a bipolar fuzzy and bipolar anti fuzzy HX subgroup and discussed some of its properties. Keywords: bipolar fuzzy HX subgroup, bipolar anti fuzzy HX subgroup, bipolar fuzzy cosets of a bipolar fuzzy HX subgroup, bipolar fuzzy middle cosets, conjugate bipolar fuzzy HX subgroup .
\end{abstract}

\section{INTRODUCTION}

The concept of fuzzy sets was initiated by Zadeh [8]. Then it has become a vigorous area of research in engineering, medical science, social science, graph theory etc. Rosenfeld [10] gave the idea of fuzzy subgroups. Li Hongxing [3] introduce the concept of HX group and the authors Luo Chengzhong, Mi Honghai , Li Hongxing [4] introduce the concept of fuzzy HX group. The author W.R.Zhang [12] commenced the concept of bipolar fuzzy sets as a generalization of fuzzy sets in 1994. In fuzzy sets the membership degree of elements range over the interval $[0,1]$. The membership degree expresses the degree of belongingness of elements to a fuzzy set. The membership degree 1 indicates that an element completely belongs to its corresponding fuzzy set and membership degree 0 indicates that an element does not belong to fuzzy set. The membership degrees on the interval $(0,1)$ indicate the partial membership to the fuzzy set. Sometimes, the membership degree means the satisfaction degree of elements to some property or constraint corresponding to a fuzzy set .In case of Bipolar-valued fuzzy sets membership degree range is enlarged from the interval $[0,1]$ to $[-1,1]$. In a bipolarvalued fuzzy set, the membership degree 0 means that the elements are irrelevant to the corresponding property, the membership degree $(0,1]$ indicates that elements somewhat satisfy the property and the membership degree $[-1,0)$ indicates that elements somewhat satisfy the implicit counter-property. R.Muthuraj et.al [6],[7] introduce the concept of bipolar fuzzy HX subgroup and bipolar anti fuzzy HX subgroup. W. B. Vasantha kandasamy [11] introduced the concept of fuzzy cosets and fuzzy middle cosets of a group. In this paper we define the concept of bipolar fuzzy cosets of a bipolar fuzzy HX subgroup and bipolar anti fuzzy HX subgroup , bipolar fuzzy middle cosets of a bipolar fuzzy HX subgroup and bipolar anti fuzzy HX subgroup and discussed some of their related properties.

\section{PRELIMINARIES}

In this section, we site the fundamental definitions that will be used in the sequel. Throughout this paper $\mathrm{G}=(\mathrm{G}, *)$ is a finite group, e is the identity element of $\mathrm{G}$, and $\mathrm{xy}$ we mean $\mathrm{x} * \mathrm{y}$.

\subsection{Definition [4]}

Let $\mathrm{G}$ be a finite group. In $2^{\mathrm{G}}-\{\phi\}$, a non-empty set $\vartheta \subset 2^{\mathrm{G}}-\{\phi\}$ is called a HX group of G, if $\vartheta$ is a group with respect to the algebraic operation defined by $\mathrm{AB}=\{\mathrm{ab} / \mathrm{a} \in \mathrm{A}$ and $\mathrm{b} \in \mathrm{B}\}$, which its unit element is denoted by $\mathrm{E}$.

\subsection{Definition [9]}

Let $\mathrm{X}$ be any non-empty set. A fuzzy subset $\mu$ of $\mathrm{X}$ is a function $\mu: \mathrm{X} \rightarrow[0,1]$.

\subsection{Definition [6],[7]}

Let $\vartheta$ be a non-empty set. A bipolar-valued fuzzy set or bipolar fuzzy set $\lambda_{\mu}$ in $\vartheta$ is an object having the form $\lambda_{\mu}=\left\{\left\langle\mathrm{A}, \lambda_{\mu}{ }^{+}(\mathrm{A}), \lambda_{\mu}{ }^{-}(\mathrm{A})\right\rangle: \mathrm{A} \in \vartheta\right\}$ where $\lambda_{\mu}{ }^{+}: \vartheta \rightarrow[0,1]$ and $\lambda_{\mu}{ }^{-}: \vartheta \rightarrow[-1,0]$ are mappings. The positive membership degree $\lambda_{\mu}{ }^{+}(\mathrm{A})$ denotes the satisfaction degree of an element $\mathrm{A}$ to the 
property corresponding to a bipolar-valued fuzzy set $\lambda_{\mu}=\left\{\left\langle\mathrm{A}, \lambda_{\mu}{ }^{+}(\mathrm{A}), \lambda_{\mu}{ }^{-}(\mathrm{A})\right\rangle\right.$ : $\left.\mathrm{A} \in \vartheta\right\}$ and the negative membership degree $\lambda_{\mu}{ }^{-}$(A) denotes the satisfaction degree of an element $\mathrm{A}$ to some implicit counter property corresponding to a bipolar-valued fuzzy set $\lambda_{\mu}=\left\{\left\langle\mathrm{A}, \lambda_{\mu}{ }^{+}(\mathrm{A}), \lambda_{\mu}{ }^{-}(\mathrm{A})\right\rangle: \mathrm{A} \in \vartheta\right\}$. If $\lambda_{\mu}{ }^{+}(\mathrm{A}) \neq 0$ and $\lambda_{\mu}{ }^{-}(\mathrm{A})=0$, it is the situation that $\mathrm{A}$ is regarded as having only positive satisfaction for $\lambda_{\mu}=\left\{\left\langle\mathrm{A}, \lambda_{\mu}{ }^{+}(\mathrm{A}), \lambda_{\mu}{ }^{-}(\mathrm{A})\right\rangle\right.$ : $\mathrm{A}$ $\in 9\}$. If $\lambda_{\mu}{ }^{+}(A)=0$ and $\lambda_{\mu}{ }^{-}(A) \neq 0$, it is the situation that $A$ does not satisfy the property of $\lambda_{\mu}=\{\langle A$, $\left.\left.\lambda_{\mu}{ }^{+}(\mathrm{A}), \lambda_{\mu}{ }^{-}(\mathrm{A})\right\rangle: \mathrm{A} \in \vartheta\right\}$, but somewhat satisfies the counter property of $\lambda_{\mu}=\left\{\left\langle\mathrm{A}, \lambda_{\mu}{ }^{+}(\mathrm{A}), \lambda_{\mu}{ }^{-}(\mathrm{A})\right\rangle: \mathrm{A} \in \vartheta\right\}$. It is possible for an element $\mathrm{A}$ to be such that $\lambda_{\mu}{ }^{+}(\mathrm{A}) \neq 0$ and $\lambda_{\mu}{ }^{-}(\mathrm{A}) \neq 0$ when the membership function of property overlaps that its counter property over some portion of $\vartheta$. For the sake of simplicity, we shall use the symbol $\lambda_{\mu}=\left(\lambda_{\mu}{ }^{+}, \lambda_{\mu}{ }^{-}\right)$for the bipolar-valued fuzzy set $\lambda_{\mu}=\left\{\left\langle\mathrm{A}, \lambda_{\mu}{ }^{+}(\mathrm{A}), \lambda_{\mu}{ }^{-}(\mathrm{A})\right\rangle\right.$ : $\left.\mathrm{A} \in \vartheta\right\}$.

\subsection{Definition [6],[7]}

Let $\mu$ be a bipolar fuzzy subset defined on G. Let $\vartheta \subset 2^{\mathrm{G}}-\{\phi\}$ be a HX group of G. A bipolar fuzzy set $\lambda_{\mu}$ defined on $\vartheta$ is said to be a bipolar fuzzy subgroup induced by $\mu$ on $\vartheta$ or a bipolar fuzzy HX subgroup of $\vartheta$ if for $A, B \in \vartheta$,

$$
\begin{array}{ll}
\text { i. } & \lambda_{\mu}{ }^{+}(\mathrm{AB}) \geq \min \left\{\lambda_{\mu}{ }^{+}(\mathrm{A}), \lambda_{\mu}{ }^{+}(\mathrm{B})\right\} \\
\text { ii. } & \lambda_{\mu}{ }^{-}(\mathrm{AB}) \leq \max \left\{\lambda_{\mu}{ }^{-}(\mathrm{A}), \lambda_{\mu}{ }^{-}(\mathrm{B})\right\} \\
\text { iii. } & \lambda_{\mu}{ }^{+}\left(\mathrm{A}^{-1}\right)=\lambda_{\mu}{ }^{+}(\mathrm{A}), \lambda_{\mu}{ }^{-}\left(\mathrm{A}^{-1}\right)=\lambda_{\mu}{ }^{-}(\mathrm{A}) .
\end{array}
$$

where

$$
\begin{aligned}
& \lambda_{\mu}{ }^{+}(\mathrm{A})=\max \left\{\mu^{+}(\mathrm{x}) / \text { for all } \mathrm{x} \in \mathrm{A} \subseteq \mathrm{G}\right\} \quad \text { and } \\
& \lambda_{\mu}{ }^{-}(\mathrm{A})=\min \left\{\mu^{-}(\mathrm{x}) / \text { for all } \mathrm{x} \in \mathrm{A} \subseteq \mathrm{G}\right\} .
\end{aligned}
$$

\subsection{Definition [6],[7]}

Let $\mu$ be a bipolar fuzzy subset defined on G. Let $\vartheta \subset 2^{\mathrm{G}}-\{\phi\}$ be a HX group of G. A bipolar fuzzy set $\lambda_{\mu}$ defined on $\vartheta$ is said to be a bipolar anti fuzzy subgroup induced by $\mu$ on $\vartheta$ or a bipolar anti fuzzy HX subgroup of $\vartheta$. if for $A, B \in \vartheta$,

$$
\begin{array}{ll}
\text { i. } & \lambda_{\mu}{ }^{+}(\mathrm{AB}) \leq \max \left\{\lambda_{\mu}{ }^{+}(\mathrm{A}), \lambda_{\mu}{ }^{+}(\mathrm{B})\right\} \\
\text { ii. } & \lambda_{\mu}{ }^{-}(\mathrm{AB}) \geq \min \left\{\lambda_{\mu}{ }^{-}(\mathrm{A}), \lambda_{\mu}{ }^{-}(\mathrm{B})\right\} \\
\text { iii. } & \lambda_{\mu}{ }^{+}\left(\mathrm{A}^{-1}\right)=\lambda_{\mu}{ }^{+}(\mathrm{A}), \lambda_{\mu}{ }^{-}\left(\mathrm{A}^{-1}\right)=\lambda_{\mu}{ }^{-}(\mathrm{A}) .
\end{array}
$$

where $\quad \lambda_{\mu}^{+}(\mathrm{A})=\min \left\{\mu^{+}(\mathrm{x}) /\right.$ for all $\left.\mathrm{x} \in \mathrm{A} \subseteq \mathrm{G}\right\}$ and

$$
\lambda_{\mu}{ }^{-}(\mathrm{A})=\max \left\{\mu^{-}(\mathrm{x}) / \text { for all } \mathrm{x} \in \mathrm{A} \subseteq \mathrm{G}\right\}
$$

\subsection{Definition}

Let $\mu=\left(\mu^{+}, \mu^{-}\right)$be a bipolar fuzzy subset of a group $\mathrm{G}$ and let $\lambda_{\mu}=\left(\lambda_{\mu}{ }^{+}, \lambda_{\mu}{ }^{-}\right)$be a bipolar fuzzy HX subgroup of a HX group $\vartheta$ with $\lambda_{\mu}{ }^{+}(\mathrm{A})=\max \left\{\mu^{+}(\mathrm{x}) /\right.$ for all $\left.\mathrm{x} \in \mathrm{A} \subseteq \mathrm{G}\right\}$ and $\lambda_{\mu}{ }^{-}(\mathrm{A})=\min \left\{\mu^{-}(\mathrm{x}) /\right.$ for all $\mathrm{x}$ $\in \mathrm{A} \subseteq \mathrm{G}\}$. For any $\mathrm{A} \in \vartheta$, the bipolar fuzzy coset $\mathrm{A} \lambda_{\mu}=\left(\mathrm{A} \lambda_{\mu}{ }^{+}, \mathrm{A} \lambda_{\mu}{ }^{-}\right)$of a bipolar fuzzy HX group $\lambda_{\mu}$ of $\vartheta$ determined by $\mathrm{A}$ is defined by

$$
\begin{array}{ll}
\text { i. } & \left(\mathrm{A} \lambda_{\mu}{ }^{+}\right)(\mathrm{X})=\lambda_{\mu}{ }^{+}\left(\mathrm{A}^{-1} \mathrm{X}\right) \\
\text { ii. } & \left(\mathrm{A} \lambda_{\mu}{ }^{-}\right)(\mathrm{X})=\lambda_{\mu}{ }^{-}\left(\mathrm{A}^{-1} \mathrm{X}\right) \text { for every } \mathrm{X} \in \vartheta .
\end{array}
$$

\subsection{Definition}

Let $\mu=\left(\mu^{+}, \mu^{-}\right)$be a bipolar fuzzy subset of a group $\mathrm{G}$ and let $\lambda_{\mu}=\left(\lambda_{\mu}{ }^{+}, \lambda_{\mu}{ }^{-}\right)$be a bipolar anti fuzzy HX subgroup of a HX group $\vartheta$ with $\lambda_{\mu}{ }^{+}(\mathrm{A})=\min \left\{\mu^{+}(\mathrm{x}) /\right.$ for all $\left.\mathrm{x} \in \mathrm{A} \subseteq \mathrm{G}\right\}$ and $\lambda_{\mu}{ }^{-}(\mathrm{A})=\max \left\{\mu^{-}(\mathrm{x}) /\right.$ for all $\mathrm{x} \in \mathrm{A} \subseteq \mathrm{G}\}$. For any $\mathrm{A} \in \vartheta$, the bipolar fuzzy coset $\mathrm{A} \lambda_{\mu}=\left(\mathrm{A} \lambda_{\mu}{ }^{+}, \mathrm{A} \lambda_{\mu}{ }^{-}\right)$of a bipolar anti fuzzy HX group $\lambda_{\mu}$ of $\vartheta$ determined by $\mathrm{A}$ is defined by

$$
\begin{array}{ll}
\text { i. } & \left(\mathrm{A} \lambda_{\mu}^{+}\right)(\mathrm{X})=\lambda_{\mu}^{+}\left(\mathrm{A}^{-1} \mathrm{X}\right) \\
\text { ii. } & \left(\mathrm{A} \lambda_{\mu}^{-}\right)(\mathrm{X})=\lambda_{\mu}{ }^{-}\left(\mathrm{A}^{-1} \mathrm{X}\right) \text { for every } \mathrm{X} \in \vartheta .
\end{array}
$$

\subsection{Example} element of $\mathrm{G}$.

Let $\mathrm{G}$ be the Klein's 4 group. Then $\mathrm{G}=\{\mathrm{e}, \mathrm{a}, \mathrm{b}, \mathrm{ab}\}$ where $\mathrm{a}^{2}=\mathrm{e}=\mathrm{b}^{2}, \mathrm{ab}=\mathrm{ba}$ and e the identity Define the bipolar fuzzy subset $\mu=\left(\mu^{+}, \mu^{-}\right)$on $\mathrm{G}$ as

$$
\begin{array}{lll}
\mu^{+}(\mathrm{x})=0.7 \text { if } \mathrm{x}=\mathrm{e}, & \mu^{+}(\mathrm{x})=0.4 \text { if } \mathrm{x}=\mathrm{a}, \quad \mu^{+}(\mathrm{x})=0.3 \text { if } \mathrm{x}=\mathrm{b}, \mathrm{ab} \\
\mu^{-}(\mathrm{x})=-0.7 \text { if } \mathrm{x}=\mathrm{e}, \quad \mu^{-}(\mathrm{x})=-0.4 \text { if } \mathrm{x}=\mathrm{a}, \mu^{-}(\mathrm{x})=-0.3 \text { if } \mathrm{x}=\mathrm{b}, \mathrm{ab}
\end{array}
$$

Let $\vartheta=\{\mathrm{E}, \mathrm{A}\}=\{\{\mathrm{e}, \mathrm{a}\},\{\mathrm{b}, \mathrm{ab}\}\}$ be a HX group on $\mathrm{G}$.

and define $\lambda_{\mu}{ }^{+}: \vartheta \rightarrow[0,1], \lambda_{\mu}{ }^{-}: \vartheta \rightarrow[-1,0]$ as $\lambda_{\mu}{ }^{+}(\mathrm{A})=\max \left\{\mu^{+}(\mathrm{x}) /\right.$ for all $\left.\mathrm{x} \in \mathrm{A} \subseteq \mathrm{G}\right\}$ and

$$
\lambda_{\mu}{ }^{-}(\mathrm{A})=\min \left\{\mu^{-}(\mathrm{x}) / \text { for all } \mathrm{x} \in \mathrm{A} \subseteq \mathrm{G}\right\}
$$




$$
\lambda_{\mu}{ }^{+}(\mathrm{X})=\left\{\begin{array}{ll}
0.7 & \text { if } \mathrm{X}=\mathrm{E} \\
0.3 & \text { if } \mathrm{X}=\mathrm{A}
\end{array} \quad \lambda_{\mu}^{-}(\mathrm{X})= \begin{cases}-0.7 & \text { if } \mathrm{X}=\mathrm{E} \\
-0.3 & \text { if } \mathrm{X}=\mathrm{A}\end{cases}\right.
$$

Clearly, $\lambda_{\mu}$ is a bipolar fuzzy HX subgroup of a HX group $\vartheta$. Now, we compute the bipolar fuzzy cosets of $\lambda_{\mu}$.

$$
\begin{aligned}
& \left(\mathrm{E} \lambda_{\mu}{ }^{+}\right)(\mathrm{X})=\left\{\begin{array}{l}
0.7 \text { if } \mathrm{X}=\mathrm{E} \\
0.3 \text { if } \mathrm{X}=\mathrm{A}
\end{array} \quad\left(\mathrm{E} \lambda_{\mu}{ }^{-}\right)(\mathrm{X})=\left\{\begin{array}{lll}
-0.7 & \text { if } \mathrm{X}=\mathrm{E} \\
-0.3 & \text { if } & \mathrm{X}=\mathrm{A}
\end{array}\right.\right. \\
& \left(\mathrm{A} \lambda_{\mu}{ }^{+}\right)(\mathrm{X})=\left\{\begin{array}{l}
0.3 \text { if } \mathrm{X}=\mathrm{E} \\
0.7 \text { if } \mathrm{X}=\mathrm{A}
\end{array} \quad\left(\mathrm{A} \lambda_{\mu}{ }^{-}\right)(\mathrm{X})= \begin{cases}-0.3 & \text { if } \mathrm{X}=\mathrm{E} \\
-0.7 & \text { if } \mathrm{X}=\mathrm{A}, \mathrm{B}\end{cases} \right.
\end{aligned}
$$

\section{Remark:}

i. If $\mathrm{A}=\mathrm{E}$, then bipolar fuzzy coset $\mathrm{A} \lambda_{\mu}=\lambda_{\mu}$

ii. If $\lambda_{\mu}$ is a bipolar fuzzy $\mathrm{HX}$ subgroup of a group $\mathrm{G}$, and $\mathrm{A}=\mathrm{E}$ then bipolar fuzzy coset $\left(A \lambda_{\mu}\right)$ is also a bipolar fuzzy HX subgroup of $\mathrm{G}$.

iii. If $\lambda_{\mu}$ is a bipolar anti fuzzy $\mathrm{HX}$ subgroup of a group $\mathrm{G}$, and $\mathrm{A}=\mathrm{E}$ then bipolar fuzzy coset $\left(\mathrm{A} \lambda_{\mu}\right)$ is also a bipolar anti fuzzy HX subgroup of $\mathrm{G}$.

\subsection{Theorem}

Let $\mu=\left(\mu^{+}, \mu^{-}\right)$be a bipolar fuzzy subset on $\mathrm{G}$ and $\lambda_{\mu}=\left(\lambda_{\mu}{ }^{+}, \lambda_{\mu}{ }^{-}\right)$be a bipolar fuzzy HX subgroup of a HX group $\vartheta$ then the bipolar fuzzy coset $\left(\mathrm{A} \lambda_{\mu}\right)=\left(\mathrm{A} \lambda_{\mu}{ }^{+}, \mathrm{A} \lambda_{\mu}{ }^{-}\right)$is a bipolar fuzzy HX subgroup of $\vartheta$ if i. $\min \left\{\lambda_{\mu}^{+}\left(\mathrm{A}^{-1} \mathrm{Y}\right), \lambda_{\mu}^{+}(\mathrm{A})\right\}=\lambda_{\mu}^{+}\left(\mathrm{A}^{-1} \mathrm{Y}\right)$

ii. $\left.\max \left\{\lambda_{\mu}^{-}\left(\mathrm{A}^{-1} \mathrm{Y}\right), \lambda_{\mu}^{-}(\mathrm{A})\right\}=\lambda_{\mu}^{-}{ }^{-} \mathrm{A}^{-1} \mathrm{Y}\right), \quad$ for every $\mathrm{Y} \in \vartheta$.

Proof : Let $\lambda_{\mu}=\left(\lambda_{\mu}{ }^{+}, \lambda_{\mu}{ }^{-}\right)$be a bipolar fuzzy HX subgroup of $\vartheta$. For every $X, Y \in \vartheta$, we have,

i. $\left(\mathrm{A} \lambda_{\mu}^{+}\right)\left(\mathrm{XY}^{-1}\right)=\lambda_{\mu}^{+}\left(\mathrm{A}^{-1} \mathrm{XY}^{-1}\right)$

$$
\begin{aligned}
& =\lambda_{\mu}^{+}\left(\mathrm{A}^{-1} \mathrm{XY}^{-1} \mathrm{AA}^{-1}\right) \\
& \geq \min \left\{\lambda_{\mu}^{+}\left(\mathrm{A}^{-1} \mathrm{X}\right), \lambda_{\mu}^{+}\left(\mathrm{Y}^{-1} \mathrm{AA}^{-1}\right)\right\} \\
& \geq \min \left\{\lambda_{\mu}^{+}\left(\mathrm{A}^{-1} \mathrm{X}\right), \min \left\{\lambda_{\mu}^{+}\left(\mathrm{Y}^{-1} \mathrm{~A}\right), \lambda_{\mu}^{+}\left(\mathrm{A}^{-1}\right)\right\}\right. \\
& \left.=\min \left\{\lambda_{\mu}^{+}\left(\mathrm{A}^{-1} \mathrm{X}\right), \min \left\{\lambda_{\mu}^{+}\left(\left(\mathrm{Y}^{-1} \mathrm{~A}\right)\right)^{-1}\right), \lambda_{\mu}^{+}(\mathrm{A})\right\}\right\} \\
& =\min \left\{\lambda_{\mu}^{+}\left(\mathrm{A}^{-1} \mathrm{X}\right), \min \left\{\lambda_{\mu}^{+}\left(\mathrm{A}^{-1} \mathrm{Y}\right), \lambda_{\mu}^{+}(\mathrm{A})\right\}\right\} \\
& =\min \left\{\lambda_{\mu}^{+}\left(\mathrm{A}^{-1} \mathrm{X}\right), \lambda_{\mu}^{+}\left(\mathrm{A}^{-1} \mathrm{Y}\right)\right\} \\
& =\min \left\{\left(\mathrm{A} \lambda_{\mu}^{+}\right)(\mathrm{X}),\left(\mathrm{A} \lambda_{\mu}^{+}\right)(\mathrm{Y})\right\}
\end{aligned}
$$

Therefore, $\left(\mathrm{A} \lambda_{\mu}^{+}\right)\left(\mathrm{XY}^{-1}\right) \geq \min \left\{\mathrm{A} \lambda_{\mu}^{+}(\mathrm{X}), \mathrm{A} \lambda_{\mu}^{+}(\mathrm{Y})\right\}$

ii. $\left(\mathrm{A} \lambda_{\mu}^{-}\right)\left(\mathrm{XY}^{-1}\right)=\lambda_{\mu}^{-}\left(\mathrm{A}^{-1} \mathrm{XY}^{-1}\right)$

$$
\begin{aligned}
& =\lambda_{\mu}{ }^{-}\left(\mathrm{A}^{-1} \mathrm{XY}^{-1} \mathrm{AA}^{-1}\right) \\
& \leq \max \left\{\lambda_{\mu}{ }^{-}\left(\mathrm{A}^{-1} \mathrm{X}\right), \lambda_{\mu}{ }^{-}\left(\mathrm{Y}^{-1} \mathrm{AA}^{-1}\right)\right\} \\
& \leq \max \left\{\lambda_{\mu}^{-}\left(\mathrm{A}^{-1} \mathrm{X}\right), \max \left\{\lambda_{\mu}^{-}\left(\mathrm{Y}^{-1} \mathrm{~A}\right), \lambda_{\mu}^{-}\left(\mathrm{A}^{-1}\right)\right\}\right. \\
& \left.=\max \left\{\lambda_{\mu}^{-}\left(\mathrm{A}^{-1} \mathrm{X}\right), \max \left\{\lambda_{\mu}^{-}\left(\left(\mathrm{Y}^{-1} \mathrm{~A}\right)\right)^{-1}\right), \lambda_{\mu}^{-}(\mathrm{A})\right\}\right\} \\
& =\max \left\{\lambda_{\mu}^{-}\left(\mathrm{A}^{-1} \mathrm{X}\right), \max \left\{\lambda_{\mu}^{-}\left(\mathrm{A}^{-1} \mathrm{Y}\right), \lambda_{\mu}^{-}(\mathrm{A})\right\}\right\} \\
& =\max \left\{\lambda_{\mu}^{-}\left(\mathrm{A}^{-1} \mathrm{X}\right), \lambda_{\mu}^{-}\left(\left(\mathrm{A}^{-1} \mathrm{Y}\right)\right\}\right. \\
& =\max \left\{\left(\mathrm{A} \lambda_{\mu}^{-}\right)(\mathrm{X}),\left(\mathrm{A} \lambda_{\mu}^{-}\right)(\mathrm{Y})\right\}
\end{aligned}
$$

Therefore, $\left(\mathrm{A} \lambda_{\mu}{ }^{-}\right)\left(\mathrm{XY}^{-1}\right) \leq \max \left\{\left(\mathrm{A} \lambda_{\mu}{ }^{-}\right)(\mathrm{X}),\left(\mathrm{A} \lambda_{\mu}{ }^{-}\right)(\mathrm{Y})\right\}$

Hence, $\mathrm{A} \lambda_{\mu}=\left(\mathrm{A} \lambda_{\mu}^{+}, \mathrm{A} \lambda_{\mu}^{-}\right)$is a bipolar fuzzy $\mathrm{HX}$ subgroup of $\vartheta$.

\subsection{Theorem}

Let $\mu=\left(\mu^{+}, \mu^{-}\right)$be a bipolar fuzzy subset on $\mathrm{G}$ and $\lambda_{\mu}=\left(\lambda_{\mu}{ }^{+}, \lambda_{\mu}{ }^{-}\right)$be a bipolar anti fuzzy HX subgroup of a HX group $\vartheta$ then the bipolar fuzzy coset $\left(\mathrm{A} \lambda_{\mu}\right)=\left(\mathrm{A} \lambda_{\mu}^{+}, \mathrm{A} \lambda_{\mu}^{-}\right)$is a bipolar anti fuzzy $\mathrm{HX}$ subgroup of $\vartheta$ if

$$
\begin{aligned}
\text { i. } \quad \max \left\{\lambda_{\mu}{ }^{+}\left(\mathrm{A}^{-1} \mathrm{Y}\right), \lambda_{\mu}{ }^{+}(\mathrm{A})\right\} & =\lambda_{\mu}{ }^{+}\left(\mathrm{A}^{-1} \mathrm{Y}\right) \\
\text { ii. } \min \left\{\lambda_{\mu}{ }^{-}\left(\mathrm{A}^{-1} \mathrm{Y}\right), \lambda_{\mu}{ }^{-}(\mathrm{A})\right\} & =\lambda_{\mu}{ }^{-}\left(\mathrm{A}^{-1} \mathrm{Y}\right), \quad \text { for every } \mathrm{Y} \in \vartheta .
\end{aligned}
$$

Proof : Let $\lambda_{\mu}=\left(\lambda_{\mu}{ }^{+}, \lambda_{\mu}{ }^{-}\right)$be a bipolar anti fuzzy HX subgroup of a HX group $\vartheta$.For every X,Y $\in \vartheta$, we have,

$$
\text { i. } \begin{aligned}
\left(\mathrm{A} \lambda_{\mu}^{+}\right)\left(\mathrm{XY}^{-1}\right) & =\lambda_{\mu}^{+}\left(\mathrm{A}^{-1} \mathrm{XY}^{-1}\right) \\
& =\lambda_{\mu}^{+}\left(\mathrm{A}^{-1} \mathrm{XY}^{-1} \mathrm{AA}^{-1}\right) \\
& \leq \max \left\{\lambda_{\mu}^{+}\left(\mathrm{A}^{-1} \mathrm{X}\right), \lambda_{\mu}^{+}\left(\mathrm{Y}^{-1} \mathrm{AA}^{-1}\right)\right\} \\
& \leq \max \left\{\lambda_{\mu}^{+}\left(\mathrm{A}^{-1} \mathrm{X}\right), \max \left\{\lambda_{\mu}^{+}\left(\mathrm{Y}^{-1} \mathrm{~A}\right), \lambda_{\mu}^{+}\left(\mathrm{A}^{-1}\right)\right\}\right. \\
& =\max \left\{\lambda_{\mu}^{+}\left(\mathrm{A}^{-1} \mathrm{X}\right), \max \left\{\lambda_{\mu}^{+}\left(\left(\mathrm{Y}^{-1} \mathrm{~A}\right)^{-1}\right), \lambda_{\mu}^{+}(\mathrm{A})\right\}\right\}
\end{aligned}
$$




$$
\begin{aligned}
& =\max \left\{\lambda_{\mu}^{+}\left(\mathrm{A}^{-1} \mathrm{X}\right), \max \left\{\lambda_{\mu}^{+}\left(\left(\mathrm{A}^{-1} \mathrm{Y}\right), \lambda_{\mu}^{+}(\mathrm{A})\right\}\right\}\right. \\
& =\max \left\{\lambda_{\mu}^{+}\left(\mathrm{A}^{-1} \mathrm{X}\right), \lambda_{\mu}^{+}\left(\left(\mathrm{A}^{-1} \mathrm{Y}\right)\right\}\right. \\
& =\max \left\{\left(\mathrm{A} \lambda_{\mu}^{+}\right)(\mathrm{X}),\left(\mathrm{A} \lambda_{\mu}^{+}\right)(\mathrm{Y})\right\}
\end{aligned}
$$

Therefore, $\left(\mathrm{A} \lambda_{\mu}{ }^{+}\right)\left(\mathrm{XY}^{-1}\right) \leq \max \left\{\left(\mathrm{A} \lambda_{\mu}{ }^{+}\right)(\mathrm{X}),\left(\mathrm{A} \lambda_{\mu}{ }^{+}\right)(\mathrm{Y})\right\}$

ii. $\left(\mathrm{A} \lambda_{\mu}{ }^{-}\right)\left(\mathrm{XY}^{-1}\right)$

$$
\begin{aligned}
& =\lambda_{\mu}{ }^{-}\left(\mathrm{A}^{-1} \mathrm{XY}^{-1}\right) \\
& =\lambda_{\mu}{ }^{-}\left(\mathrm{A}^{-1} \mathrm{XY}^{-1} \mathrm{AA}^{-1}\right) \\
& \geq \min \left\{\lambda_{\mu}{ }^{-}\left(\mathrm{A}^{-1} \mathrm{X}\right), \lambda_{\mu}{ }^{-}\left(\mathrm{Y}^{-1} \mathrm{AA}^{-1}\right)\right\} \\
& \geq \min \left\{\lambda_{\mu}^{-}\left(\mathrm{A}^{-1} \mathrm{X}\right), \min \left\{\lambda_{\mu}^{-}\left(\mathrm{Y}^{-1} \mathrm{~A}\right), \lambda_{\mu}{ }^{-}\left(\mathrm{A}^{-1}\right)\right\}\right. \\
& =\min \left\{\lambda_{\mu}{ }^{-}\left(\mathrm{A}^{-1} \mathrm{X}\right), \min \left\{\lambda_{\mu}^{-}\left(\left(\mathrm{Y}^{-1} \mathrm{~A}\right)^{-1}\right), \lambda_{\mu}{ }^{-}(\mathrm{A})\right\}\right\} \\
& =\min \left\{\lambda_{\mu}^{-}\left(\mathrm{A}^{-1} \mathrm{X}\right), \min \left\{\lambda_{\mu}^{-}\left(\left(\mathrm{A}^{-1} \mathrm{Y}\right), \lambda_{\mu}{ }^{-}(\mathrm{A})\right\}\right\}\right. \\
& =\min \left\{\lambda_{\mu}^{-}\left(\mathrm{A}^{-1} \mathrm{X}\right), \lambda_{\mu}^{-}\left(\left(\mathrm{A}^{-1} \mathrm{Y}\right)\right\}\right. \\
& =\min \left\{\left(\mathrm{A} \lambda_{\mu}^{-}\right)(\mathrm{X}),\left(\mathrm{A} \lambda_{\mu}^{-}\right)(\mathrm{Y})\right\}
\end{aligned}
$$

Therefore, $\left(\mathrm{A} \lambda_{\mu}^{-}\right)\left(\mathrm{XY}^{-1}\right) \geq \min \left\{\left(\mathrm{A} \lambda_{\mu}^{-}\right)(\mathrm{X}),\left(\mathrm{A} \lambda_{\mu}{ }^{-}\right)(\mathrm{Y})\right\}$

Hence, $\mathrm{A} \lambda_{\mu}=\left(\mathrm{A} \lambda_{\mu}{ }^{+}, \mathrm{A} \lambda_{\mu}{ }^{-}\right)$is a bipolar anti fuzzy HX subgroup of $\vartheta$.

\subsection{Theorem}

Let $\mu=\left(\mu^{+}, \mu^{-}\right)$be a bipolar fuzzy subset on $\mathrm{G}$ and $\lambda_{\mu}=\left(\lambda_{\mu}{ }^{+}, \lambda_{\mu}{ }^{-}\right)$be a bipolar fuzzy HX subgroup of a HX group $\vartheta$ then $\mathrm{X} \lambda_{\mu}=\mathrm{Y} \lambda_{\mu}$, for $\mathrm{X}, \mathrm{Y} \in \vartheta$ if and only if

$$
\begin{array}{rlrl} 
& \text { i. } & \lambda_{\mu}{ }^{+}\left(\mathrm{X}^{-1} \mathrm{Y}\right) & =\lambda_{\mu}{ }^{+}\left(\mathrm{Y}^{-1} \mathrm{X}\right)=\lambda_{\mu}{ }^{+}(\mathrm{E}) \\
\text { ii. } & \lambda_{\mu}{ }^{-}\left(\mathrm{X}^{-1} \mathrm{Y}\right) & =\lambda_{\mu}{ }^{-}\left(\mathrm{Y}^{-1} \mathrm{X}\right)=\lambda_{\mu}{ }^{-}(\mathrm{E})
\end{array}
$$

Proof : Let $\lambda_{\mu}=\left(\lambda_{\mu}{ }^{+}, \lambda_{\mu}{ }^{-}\right)$be a bipolar fuzzy HX subgroup of a HX group $\vartheta$.
Let $\mathrm{X} \lambda_{\mu}=\mathrm{Y} \lambda_{\mu}$, for $\mathrm{X}, \mathrm{Y} \in \vartheta$, that is i. $\mathrm{X} \lambda_{\mu}{ }^{+}=\mathrm{Y} \lambda_{\mu}{ }^{+}$
ii. $\mathrm{X} \lambda_{\mu}{ }^{-}=\mathrm{Y} \lambda_{\mu}{ }^{-}$

Then, $\left(\mathrm{X} \lambda_{\mu}{ }^{+}\right)(\mathrm{X})=\left(\mathrm{Y} \lambda_{\mu}{ }^{+}\right)(\mathrm{X}),\left(\mathrm{X} \lambda_{\mu}{ }^{+}\right)(\mathrm{Y})=\left(\mathrm{Y} \lambda_{\mu}{ }^{+}\right)(\mathrm{Y})$ and

$$
\begin{aligned}
& \left(\mathrm{X} \lambda_{\mu}{ }^{-}\right)(\mathrm{X})=\left(\mathrm{Y} \lambda_{\mu}^{-}\right)(\mathrm{X}),\left(\mathrm{X} \lambda_{\mu}^{-}\right)(\mathrm{Y})=\left(\mathrm{Y} \lambda_{\mu}^{-}\right)(\mathrm{Y}) \text { which implies that, } \\
& \lambda_{\mu}^{+}\left(\mathrm{X}^{-1} \mathrm{X}\right)=\lambda_{\mu}{ }^{+}\left(\mathrm{Y}^{-1} \mathrm{X}\right), \lambda_{\mu}^{+}\left(\mathrm{X}^{-1} \mathrm{Y}\right)=\lambda_{\mu}^{+}\left(\mathrm{Y}^{-1} \mathrm{Y}\right) \text { and } \\
& \lambda_{\mu}{ }^{-}\left(\mathrm{X}^{-1} \mathrm{X}\right)=\lambda_{\mu}^{-}\left(\mathrm{Y}^{-1} \mathrm{X}\right), \lambda_{\mu}{ }^{-}\left(\mathrm{X}^{-1} \mathrm{Y}\right)=\lambda_{\mu}^{-}\left(\mathrm{Y}^{-1} \mathrm{Y}\right) \quad(\text { By definition })
\end{aligned}
$$

$\Rightarrow \lambda_{\mu}{ }^{+}(\mathrm{E})=\lambda_{\mu}{ }^{+}\left(\mathrm{Y}^{-1} \mathrm{X}\right), \lambda_{\mu}{ }^{+}\left(\mathrm{X}^{-1} \mathrm{Y}\right)=\lambda_{\mu}{ }^{+}(\mathrm{E})$ and

$\lambda_{\mu}{ }^{-}(\mathrm{E})=\lambda_{\mu}{ }^{-}\left(\mathrm{Y}^{-1} \mathrm{X}\right), \lambda_{\mu}{ }^{-}\left(\mathrm{X}^{-1} \mathrm{Y}\right)=\lambda_{\mu}{ }^{-}(\mathrm{E})$.

Hence, $\lambda_{\mu}{ }^{+}\left(\mathrm{X}^{-1} \mathrm{Y}\right)=\lambda_{\mu}{ }^{+}\left(\mathrm{Y}^{-1} \mathrm{X}\right)=\lambda_{\mu}{ }^{+}(\mathrm{E})$ and $\lambda_{\mu}{ }^{-}\left(\mathrm{X}^{-1} \mathrm{Y}\right)=\lambda_{\mu}{ }^{-}\left(\mathrm{Y}^{-1} \mathrm{X}\right)=\lambda_{\mu}{ }^{-}(\mathrm{E})$.

Conversely,

For $\mathrm{X}, \mathrm{Y} \in \vartheta$. Let $\lambda_{\mu}{ }^{+}\left(\mathrm{X}^{-1} \mathrm{Y}\right)=\lambda_{\mu}{ }^{+}\left(\mathrm{Y}^{-1} \mathrm{X}\right)=\lambda_{\mu}{ }^{+}(\mathrm{E})$ and $\lambda_{\mu}{ }^{-}\left(\mathrm{X}^{-1} \mathrm{Y}\right)=\lambda_{\mu}{ }^{-}\left(\mathrm{Y}^{-1} \mathrm{X}\right)=\lambda_{\mu}{ }^{-}(\mathrm{E})$,

For every $\mathrm{U} \in \vartheta$, we have,

$$
\text { i. } \begin{aligned}
\left(\mathrm{X} \lambda_{\mu}{ }^{+}\right)(\mathrm{U}) & =\lambda_{\mu}^{+}\left(\mathrm{X}^{-1} \mathrm{U}\right) \\
& =\lambda_{\mu}^{+}\left(\mathrm{X}^{-1} \mathrm{YY}^{-1} \mathrm{U}\right) \\
& \geq \min \left\{\lambda_{\mu}^{+}\left(\mathrm{X}^{-1} \mathrm{Y}\right), \lambda_{\mu}^{+}\left(\mathrm{Y}^{-1} \mathrm{U}\right)\right\} \\
& =\min \left\{\lambda_{\mu}^{+}(\mathrm{E}), \lambda_{\mu}^{+}\left(\mathrm{Y}^{-1} \mathrm{U}\right)\right\} \\
& =\lambda_{\mu}^{+}\left(\mathrm{Y}^{-1} \mathrm{U}\right) \\
& =\left(\mathrm{Y} \lambda_{\mu}^{+}\right)(\mathrm{U}) .
\end{aligned}
$$

Therefore, $\left(\mathrm{X} \lambda_{\mu}{ }^{+}\right)(\mathrm{U}) \geq\left(\mathrm{Y} \lambda_{\mu}{ }^{+}\right)(\mathrm{U})$.

similarly, $\left(\mathrm{Y} \lambda_{\mu}^{+}\right)(\mathrm{U}) \geq\left(\mathrm{X} \lambda_{\mu}^{+}\right)(\mathrm{U})$.

Hence, $\quad\left(\mathrm{X} \lambda_{\mu}{ }^{+}\right)(\mathrm{U})=\left(\mathrm{Y} \lambda_{\mu}{ }^{+}\right)(\mathrm{U})$.

ii. $\left(X \lambda_{\mu}^{-}\right)(\mathrm{U})$

$$
\begin{aligned}
& =\lambda_{\mu}^{-}\left(\mathrm{X}^{-1} \mathrm{U}\right) \\
& =\lambda_{\mu}^{-}\left(\mathrm{X}^{-1} \mathrm{YY}^{-1} \mathrm{U}\right) \\
& \leq \max \left\{\lambda_{\mu}{ }^{-}\left(\mathrm{X}^{-1} \mathrm{Y}\right), \lambda_{\mu}^{-}\left(\mathrm{Y}^{-1} \mathrm{U}\right)\right\} \\
& =\max \left\{\lambda_{\mu}^{-}(\mathrm{E}), \lambda_{\mu}^{-}\left(\mathrm{Y}^{-1} \mathrm{U}\right)\right\} \\
& =\lambda_{\mu}^{-}\left(\mathrm{Y}^{-1} \mathrm{U}\right) \\
& =\mathrm{Y}^{-}(\mathrm{U}) .
\end{aligned}
$$

Therefore, $\left(\mathrm{X} \lambda_{\mu}{ }^{-}\right)(\mathrm{U}) \leq\left(\mathrm{Y} \lambda_{\mu}{ }^{-}\right)(\mathrm{U})$.

similarly, $\left(\mathrm{Y} \lambda_{\mu}^{-}\right)(\mathrm{U}) \leq\left(\mathrm{X} \lambda_{\mu}^{-}\right)(\mathrm{U})$.

Hence, $\quad\left(\mathrm{X} \lambda_{\mu}{ }^{-}\right)(\mathrm{U})=\left(\mathrm{Y} \lambda_{\mu}{ }^{-}\right)(\mathrm{U})$.

Hence, $\mathrm{X} \lambda_{\mu}=\mathrm{Y} \lambda_{\mu}$, for $\mathrm{X}, \mathrm{Y} \in \vartheta$. 


\subsection{Theorem}

Let $\mu=\left(\mu^{+}, \mu^{-}\right)$be a bipolar fuzzy subset on $\mathrm{G}$ and $\lambda_{\mu}=\left(\lambda_{\mu}{ }^{+}, \lambda_{\mu}{ }^{-}\right)$be a bipolar anti fuzzy HX subgroup of a HX group $\vartheta$ then $X \lambda_{\mu}=Y \lambda_{\mu}$, for $X, Y \in \vartheta$ if and only if

$$
\begin{array}{ll}
\text { i. } & \lambda_{\mu}{ }^{+}\left(\mathrm{X}^{-1} \mathrm{Y}\right)=\lambda_{\mu}{ }^{+}\left(\mathrm{Y}^{-1} \mathrm{X}\right)=\lambda_{\mu}{ }^{+}(\mathrm{E}) \\
\text { ii. } & \lambda_{\mu}{ }^{-}\left(\mathrm{X}^{-1} \mathrm{Y}\right)=\lambda_{\mu}{ }^{-}\left(\mathrm{Y}^{-1} \mathrm{X}\right)=\lambda_{\mu}{ }^{-}(\mathrm{E})
\end{array}
$$

Proof : Let $\lambda_{\mu}=\left(\lambda_{\mu}{ }^{+}, \lambda_{\mu}{ }^{-}\right)$be a bipolar anti fuzzy HX subgroup of a HX group $\vartheta$.

Let $\mathrm{X} \lambda_{\mu}=\mathrm{Y} \lambda_{\mu}$, for $\mathrm{x}, \mathrm{y} \in \mathrm{G}$ that is i. $\mathrm{X} \lambda_{\mu}{ }^{+}=\mathrm{Y} \lambda_{\mu}{ }^{+}$

Then, $\left(\mathrm{X} \lambda_{\mu}^{+}\right)(\mathrm{X})=\left(\mathrm{Y} \lambda_{\mu}^{+}\right)(\mathrm{X}),\left(\mathrm{X} \lambda_{\mu}{ }^{+}\right)(\mathrm{Y})=\left(\mathrm{Y} \lambda_{\mu}{ }^{+}\right)(\mathrm{Y})$ and

ii. $\mathrm{X} \lambda_{\mu}{ }^{-}=\mathrm{Y} \lambda_{\mu}{ }^{-}$

$\left(\mathrm{X} \lambda_{\mu}^{-}\right)(\mathrm{X})=\left(\mathrm{Y} \lambda_{\mu}^{-}\right)(\mathrm{X}), \quad\left(\mathrm{X} \lambda_{\mu}^{-}\right)(\mathrm{Y})=\left(\mathrm{Y} \lambda_{\mu}^{-}\right)(\mathrm{Y})$ which implies that,

$\lambda_{\mu}{ }^{+}\left(\mathrm{X}^{-1} \mathrm{X}\right)=\lambda_{\mu}{ }^{+}\left(\mathrm{Y}^{-1} \mathrm{X}\right), \lambda_{\mu}^{+}\left(\mathrm{X}^{-1} \mathrm{Y}\right)=\lambda_{\mu}^{+}\left(\mathrm{Y}^{-1} \mathrm{Y}\right)$ and

$\lambda_{\mu}^{-}\left(\mathrm{X}^{-1} \mathrm{X}\right)=\lambda_{\mu}^{-}\left(\mathrm{Y}^{-1} \mathrm{X}\right), \lambda_{\mu}^{-}\left(\mathrm{X}^{-1} \mathrm{Y}\right)=\lambda_{\mu}^{-}\left(\mathrm{Y}^{-1} \mathrm{Y}\right)$ ( By definition of bipolar fuzzy coset $)$

$\Rightarrow \lambda_{\mu}{ }^{+}(\mathrm{E})=\lambda_{\mu}{ }^{+}\left(\mathrm{Y}^{-1} \mathrm{X}\right), \lambda_{\mu}{ }^{+}\left(\mathrm{X}^{-1} \mathrm{Y}\right)=\lambda_{\mu}{ }^{+}(\mathrm{E})$ and

$\lambda_{\mu}{ }^{-}(\mathrm{E})=\lambda_{\mu}{ }^{-}\left(\mathrm{Y}^{-1} \mathrm{X}\right) \quad, \lambda_{\mu}^{-}\left(\mathrm{X}^{-1} \mathrm{Y}\right)=\lambda_{\mu}{ }^{-}(\mathrm{E})$.

Hence, $\lambda_{\mu}{ }^{+}\left(\mathrm{X}^{-1} \mathrm{Y}\right)=\lambda_{\mu}{ }^{+}\left(\mathrm{Y}^{-1} \mathrm{X}\right)=\lambda_{\mu}{ }^{+}(\mathrm{E})$ and $\lambda_{\mu}{ }^{-}\left(\mathrm{X}^{-1} \mathrm{Y}\right)=\lambda_{\mu}{ }^{-}\left(\mathrm{Y}^{-1} \mathrm{X}\right)=\lambda_{\mu}{ }^{-}(\mathrm{E})$.

Conversely,

For $\mathrm{X}, \mathrm{Y} \in$ ९.Let $\lambda_{\mu}{ }^{+}\left(\mathrm{X}^{-1} \mathrm{Y}\right)=\lambda_{\mu}{ }^{+}\left(\mathrm{Y}^{-1} \mathrm{X}\right)=\lambda_{\mu}{ }^{+}(\mathrm{E})$ and $\lambda_{\mu}{ }^{-}\left(\mathrm{X}^{-1} \mathrm{Y}\right)=\lambda_{\mu}{ }^{-}\left(\mathrm{Y}^{-1} \mathrm{X}\right)=\lambda_{\mu}{ }^{-}(\mathrm{E})$,

For every $\mathrm{U} \in \vartheta$, we have,

$$
\text { i. } \begin{aligned}
\left(\mathrm{X} \lambda_{\mu}{ }^{+}\right)(\mathrm{U}) & =\lambda_{\mu}^{+}\left(\mathrm{X}^{-1} \mathrm{U}\right) \\
& =\lambda_{\mu}^{+}\left(\mathrm{X}^{-1} \mathrm{YY}^{-1} \mathrm{U}\right) \\
& \leq \max \left\{\lambda_{\mu}^{+}\left(\mathrm{X}^{-1} \mathrm{Y}\right), \lambda_{\mu}^{+}\left(\mathrm{Y}^{-1} \mathrm{U}\right)\right\} \\
& =\max \left\{\lambda_{\mu}^{+}(\mathrm{E}), \lambda_{\mu}^{+}\left(\mathrm{Y}^{-1} \mathrm{U}\right)\right\} \\
& =\lambda_{\mu}^{+}\left(\mathrm{Y}^{-1} \mathrm{U}\right) \\
& =\left(\mathrm{Y} \lambda_{\mu}^{+}\right)(\mathrm{U}) .
\end{aligned}
$$

Therefore, $\left(\mathrm{X} \lambda_{\mu}{ }^{+}\right)(\mathrm{U}) \leq\left(\mathrm{Y} \lambda_{\mu}{ }^{+}\right)(\mathrm{U})$.

similarly, $\left(\mathrm{Y} \lambda_{\mu}^{+}\right)(\mathrm{U}) \leq\left(\mathrm{X} \lambda_{\mu}^{+}\right)(\mathrm{U})$.

Hence, $\left(\mathrm{X} \lambda_{\mu}^{+}\right)(\mathrm{U})=\left(\mathrm{Y} \lambda_{\mu}^{+}\right)(\mathrm{U})$.

ii. $\left(\mathrm{X} \lambda_{\mu}{ }^{-}\right)(\mathrm{U}) \quad=\lambda_{\mu}{ }^{-}\left(\mathrm{X}^{-1} \mathrm{U}\right)$

$=\lambda_{\mu}^{-}\left(\mathrm{X}^{-1} \mathrm{YY}^{-1} \mathrm{U}\right)$

$\geq \min \left\{\lambda_{\mu}{ }^{-}\left(\mathrm{X}^{-1} \mathrm{Y}\right), \lambda_{\mu}^{-}\left(\mathrm{Y}^{-1} \mathrm{U}\right)\right\}$

$=\min \left\{\lambda_{\mu}^{-}(\mathrm{E}), \lambda_{\mu}{ }^{-}\left(\mathrm{Y}^{-1} \mathrm{U}\right)\right\}$

$=\lambda_{\mu}{ }^{-}\left(\mathrm{Y}^{-1} \mathrm{U}\right)$

$=\left(\mathrm{Y} \lambda_{\mu}{ }^{-}\right)(\mathrm{U})$.

Therefore, $\left(\mathrm{X} \lambda_{\mu}{ }^{-}\right)(\mathrm{U}) \geq\left(\mathrm{Y} \lambda_{\mu}{ }^{-}\right)(\mathrm{U})$.

similarly, $\left(\mathrm{Y} \lambda_{\mu}^{-}\right)(\mathrm{U}) \geq\left(\mathrm{X} \lambda_{\mu}^{-}\right)(\mathrm{U})$.

Hence, $\left(\mathrm{X} \lambda_{\mu}{ }^{-}\right)(\mathrm{U})=\left(\mathrm{Y} \lambda_{\mu}{ }^{-}\right)(\mathrm{U})$.

Hence, $\mathrm{X} \lambda_{\mu}=\mathrm{Y} \lambda_{\mu}$, for $\mathrm{X}, \mathrm{Y} \in \vartheta$.

\subsection{Theorem}

Let $\mu=\left(\mu^{+}, \mu^{-}\right)$be a bipolar fuzzy subset on $G$ and $\lambda_{\mu}=\left(\lambda_{\mu}{ }^{+}, \lambda_{\mu}{ }^{-}\right)$be a bipolar fuzzy HX subgroup of $\vartheta$ and $\mathrm{X} \lambda_{\mu}=\mathrm{Y} \lambda_{\mu}$, for $\mathrm{X}, \mathrm{Y} \in \vartheta$ then $\lambda_{\mu}(\mathrm{X})=\lambda_{\mu}(\mathrm{Y})$.

Proof : Let $\lambda_{\mu}=\left(\lambda_{\mu}{ }^{+}, \lambda_{\mu}{ }^{-}\right)$be a bipolar fuzzy HX subgroup of $\vartheta$ and $\mathrm{X} \lambda_{\mu}=\mathrm{Y} \lambda_{\mu}$, for $\mathrm{X}, \mathrm{Y} \in \vartheta$.

Now, i. $\quad \lambda_{\mu}^{+}(\mathrm{X})=\lambda_{\mu}^{+}\left(\mathrm{YY}^{-1} \mathrm{X}\right)$

$$
\begin{aligned}
& \geq \min \left\{\lambda_{\mu}{ }^{+}(\mathrm{Y}), \lambda_{\mu}{ }^{+}\left(\mathrm{Y}^{-1} \mathrm{X}\right)\right\} \\
& =\min \left\{\lambda_{\mu}^{+}(\mathrm{Y}), \lambda_{\mu}^{+}(\mathrm{E})\right\}, \text { by Theorem } 2.3 \\
& =\lambda_{\mu}{ }^{+}(\mathrm{Y}) .
\end{aligned}
$$

Therefore, $\quad \lambda_{\mu}{ }^{+}(\mathrm{X}) \geq \lambda_{\mu}{ }^{+}(\mathrm{Y})$.

Similarly, $\quad \lambda_{\mu}{ }^{+}(\mathrm{Y}) \geq \lambda_{\mu}{ }^{+}(\mathrm{X})$.

Hence, $\quad \lambda_{\mu}{ }^{+}(\mathrm{X})=\lambda_{\mu}{ }^{+}(\mathrm{Y})$.

ii. $\quad \lambda_{\mu}{ }^{-}(\mathrm{X})=\lambda_{\mu}{ }^{-}\left(\mathrm{YY}^{-1} \mathrm{X}\right)$

$$
\begin{aligned}
& \leq \max \left\{\lambda_{\mu}^{-}(\mathrm{Y}), \lambda_{\mu}{ }^{-}\left(\mathrm{Y}^{-1} \mathrm{X}\right)\right\} \\
& =\max \left\{\lambda_{\mu}{ }^{-}(\mathrm{Y}), \lambda_{\mu}{ }^{-}(\mathrm{E})\right\}, \text { by Theorem } 2.3 \\
& =\lambda_{\mu}{ }^{-}(\mathrm{Y}) .
\end{aligned}
$$

Therefore, $\quad \lambda_{\mu}{ }^{-}(\mathrm{X}) \leq \lambda_{\mu}{ }^{-}(\mathrm{Y})$.

Similarly, $\quad \lambda_{\mu}^{-}(\mathrm{Y}) \leq \lambda_{\mu}^{-}(\mathrm{X})$.

Hence, $\quad \lambda_{\mu}{ }^{-}(\mathrm{X})=\lambda_{\mu}{ }^{-}(\mathrm{Y})$.

Hence, $\quad \lambda_{\mu}(\mathrm{X})=\lambda_{\mu}(\mathrm{Y})$. 


\subsection{Theorem}

Let $\mu=\left(\mu^{+}, \mu^{-}\right)$be a bipolar fuzzy subset on $\mathrm{G}$ and $\lambda_{\mu}=\left(\lambda_{\mu}{ }^{+}, \lambda_{\mu}{ }^{-}\right)$be a bipolar anti fuzzy subgroup of $\vartheta$ and $\mathrm{X} \lambda_{\mu}=\mathrm{Y} \lambda_{\mu}$, for $\mathrm{X}, \mathrm{Y} \in \vartheta$ then $\lambda_{\mu}(\mathrm{X})=\lambda_{\mu}(\mathrm{Y})$.

Proof : Let $\lambda_{\mu}=\left(\lambda_{\mu}{ }^{+}, \lambda_{\mu}{ }^{-}\right)$be a bipolar anti fuzzy HX subgroup of $\vartheta$ and $\mathrm{X} \lambda_{\mu}=\mathrm{Y} \lambda_{\mu}$, for $\mathrm{X}, \mathrm{Y} \in \vartheta$.

Now, i. $\quad \lambda_{\mu}{ }^{+}(\mathrm{X})=\lambda_{\mu}{ }^{+}\left(\mathrm{YY}^{-1} \mathrm{X}\right)$

$$
\begin{aligned}
& \leq \max \left\{\lambda_{\mu}{ }^{+}(\mathrm{Y}), \lambda_{\mu}{ }^{+}\left(\mathrm{Y}^{-1} \mathrm{X}\right)\right\} \\
& =\max \left\{\lambda_{\mu}{ }^{+}(\mathrm{Y}), \lambda_{\mu}{ }^{+}(\mathrm{E})\right\}, \text { by Theorem } 2.3 \\
& =\lambda_{\mu}{ }^{+}(\mathrm{Y}) .
\end{aligned}
$$

Therefore, $\lambda_{\mu}^{+}(\mathrm{X}) \leq \lambda_{\mu}^{+}(\mathrm{Y})$.

Similarly, $\quad \lambda_{\mu}{ }^{+}(\mathrm{Y}) \leq \lambda_{\mu}^{+}(\mathrm{X})$.

Hence, $\quad \lambda_{\mu}^{+}(\mathrm{X})=\lambda_{\mu}{ }^{+}(\mathrm{Y})$.

ii. $\quad \lambda_{\mu}{ }^{-}(\mathrm{X})=\lambda_{\mu}{ }^{-}\left(\mathrm{YY}^{-1} \mathrm{X}\right)$

$\geq \min \left\{\lambda_{\mu}^{-}(\mathrm{Y}), \lambda_{\mu}^{-}\left(\mathrm{Y}^{-1} \mathrm{X}\right)\right\}$

$=\min \left\{\lambda_{\mu}{ }^{-}(\mathrm{Y}), \lambda_{\mu}{ }^{-}(\mathrm{E})\right\}$, by Theorem 2.3

$=\lambda_{\mu}^{-}(\mathrm{Y})$.

Therefore, $\quad \lambda_{\mu}^{-}(\mathrm{X}) \geq \lambda_{\mu}^{-}(\mathrm{Y})$.

Similarly, $\quad \lambda_{\mu}^{-}(\mathrm{Y}) \geq \lambda_{\mu}^{-}(\mathrm{X})$.

Hence, $\quad \lambda_{\mu}^{-}(\mathrm{X})=\lambda_{\mu}{ }^{-}(\mathrm{Y})$.

Hence, $\lambda_{\mu}(\mathrm{X})=\lambda_{\mu}(\mathrm{Y})$.

\subsection{Definition}

Let $\mu=\left(\mu^{+}, \mu^{-}\right)$be a bipolar fuzzy subset on $\mathrm{G}$ and $\lambda_{\mu}=\left(\lambda_{\mu}{ }^{+}, \lambda_{\mu}{ }^{-}\right)$be a bipolar fuzzy HX subgroup ( bipolar anti fuzzy HX subgroup) of a HX group $\vartheta$. Then for any $A, B \in \vartheta$, a bipolar fuzzy middle coset $\mathrm{A} \lambda_{\mu} \mathrm{B}=\left(\mathrm{A} \lambda_{\mu}{ }^{+} \mathrm{B}, \mathrm{A} \lambda_{\mu}{ }^{-} \mathrm{B}\right)$ of a bipolar fuzzy HX subgroup (bipolar anti fuzzy HX subgroup) $\lambda_{\mu}$ of $\vartheta$ determined by $\mathrm{A}$ and $\mathrm{B}$ is defined as

$$
\begin{aligned}
& \text { i. } \quad\left(\mathrm{A} \lambda_{\mu}{ }^{+} \mathrm{B}\right)(\mathrm{X})=\lambda_{\mu}{ }^{+}\left(\mathrm{A}^{-1} \mathrm{XB}^{-1}\right) \\
& \text { ii. } \quad\left(\mathrm{A} \lambda_{\mu}{ }^{-} \mathrm{B}\right)(\mathrm{X})=\lambda_{\mu}{ }^{-}\left(\mathrm{A}^{-1} \mathrm{X} \mathrm{B}^{-1}\right) .
\end{aligned}
$$

\subsection{Example}

Let $G=\left\{Z_{7}-\{0\}, \bullet_{7}\right\}$ be a group with respect to multiplication. Define the bipolar fuzzy subset $\mu=\left(\mu^{+}, \mu^{-}\right)$on $G$ as

$$
\mu^{+}(x)=\left\{\begin{array}{ll}
0.8 & \text { if } x=1 \\
0.5 & \text { if } x=2,4 \\
0.4 & \text { if } x=3,5 \\
0.3 & \text { if } x=6
\end{array} \quad \mu^{-}(x)= \begin{cases}-0.8 & \text { if } x=1 \\
-0.5 & \text { if } x=2,4 \\
-0.4 & \text { if } x=3,5 \\
-0.3 & \text { if } x=6\end{cases}\right.
$$

Clearly $\mu=\left(\mu^{+}, \mu^{-}\right)$is a bipolar fuzzy subgroup of G. Let $\vartheta=\{\mathrm{E}, \mathrm{A}, \mathrm{B}\}$, where $\mathrm{E}=\{1,6\}, \mathrm{A}=\{2,5\}, \mathrm{B}=\{3,4\}$. Clearly $\left(\vartheta, \bullet_{7}\right)$ is a bipolar fuzzy HX subgroup.

Then the bipolar fuzzy middle coset $\left(\mathrm{A} \lambda_{\mu} \mathrm{B}\right)=\left(\mathrm{A} \lambda_{\mu}{ }^{+} \mathrm{B}, \mathrm{A} \lambda_{\mu}{ }^{-} \mathrm{B}\right)$ is defined as

$$
\left(\mathrm{A} \lambda_{\mu}{ }^{+} \mathrm{B}\right)(\mathrm{X})=\left\{\begin{array}{l}
0.8 \text { if } \mathrm{X}=\mathrm{E} \\
0.5 \text { if } \mathrm{X}=\mathrm{A}, \mathrm{B}
\end{array} \quad\left(\mathrm{A} \lambda_{\mu}{ }^{-} \mathrm{B}\right)(\mathrm{X})= \begin{cases}-0.8 & \text { if } \mathrm{X}=\mathrm{E} \\
-0.5 & \text { if } \mathrm{X}=\mathrm{A}, \mathrm{B}\end{cases}\right.
$$

Clearly $\left(\mathrm{A} \lambda_{\mu} \mathrm{B}\right)$ is a bipolar fuzzy $\mathrm{HX}$ subgroup of $\vartheta$ since $\mathrm{B}=\mathrm{A}^{-1}$, But

$$
\left(\mathrm{E} \lambda_{\mu}{ }^{+} \mathrm{A}\right)(\mathrm{X})=\left\{\begin{array}{ll}
0.5 & \text { if } \mathrm{X}=\mathrm{E} \\
0.8 & \text { if } \mathrm{X}=\mathrm{A}, \mathrm{B}
\end{array} \quad\left(\mathrm{A} \lambda_{\mu}{ }^{-} \mathrm{B}\right)(\mathrm{X})=\left\{\begin{array}{l}
-0.5 \text { if } \mathrm{X}=\mathrm{E} \\
-0.8 \text { if } \mathrm{X}=\mathrm{A}, \mathrm{B}
\end{array}\right.\right.
$$

The bipolar fuzzy middle coset $\left(\mathrm{E} \lambda_{\mu} \mathrm{A}\right)$ is not a bipolar fuzzy HX subgroup of $\vartheta$.

\section{Remark:}

i. Let $\lambda_{\mu}=\left(\lambda_{\mu}{ }^{+}, \lambda_{\mu}{ }^{-}\right)$be a bipolar fuzzy HX subgroup of $\vartheta$, then bipolar fuzzy middle coset $\left(A \lambda_{\mu} B\right)=\left(A \lambda_{\mu}{ }^{+} B, A \lambda_{\mu}{ }^{-} B\right)$ is also a bipolar fuzzy HX subgroup of $\vartheta$ if $B=A^{-1}$.

ii. Let $\lambda_{\mu}=\left(\lambda_{\mu}{ }^{+}, \lambda_{\mu}{ }^{-}\right)$be a bipolar anti fuzzy HX subgroup of $\vartheta$, then bipolar fuzzy middle coset 
$\left(\mathrm{A} \lambda_{\mu} \mathrm{B}\right)=\left(\mathrm{A} \lambda_{\mu}{ }^{+} \mathrm{B}, \mathrm{A} \lambda_{\mu}{ }^{-} \mathrm{B}\right)$ is also a bipolar anti fuzzy HX subgroup of $\vartheta$ if $\mathrm{B}=\mathrm{A}^{-1}$.

\subsection{Theorem}

Let $\mu=\left(\mu^{+}, \mu^{-}\right)$be a bipolar fuzzy subset on $\mathrm{G}$ and $\lambda_{\mu}=\left(\lambda_{\mu^{+}}{ }^{+}, \lambda_{\mu}{ }^{-}\right)$be a bipolar fuzzy HX subgroup of $\vartheta$ then the bipolar middle $\operatorname{coset}\left(A \lambda_{\mu} A^{-1}\right)=\left(A \lambda_{\mu}{ }^{+} A^{-1}, A \lambda_{\mu}{ }^{-} A^{-1}\right)$ is a bipolar fuzzy HX subgroup of $\vartheta$.

Proof : Let $\lambda_{\mu}=\left(\lambda_{\mu}{ }^{+}, \lambda_{\mu}{ }^{-}\right)$be a bipolar fuzzy HX subgroup of $\vartheta$ and Let for some A $\in \vartheta$, for every X,Y $\in \vartheta$ then

$$
\text { i. } \begin{aligned}
\left(\mathrm{A} \lambda_{\mu}{ }^{+} \mathrm{A}^{-1}\right)\left(\mathrm{XY}^{-1}\right) \quad & =\lambda_{\mu}^{+}\left(\mathrm{A}^{-1} \mathrm{XY} \mathrm{Y}^{-1} \mathrm{~A}\right), \text { by the definition } \\
& =\lambda_{\mu}^{+}\left(\mathrm{A}^{-1} \mathrm{X} \mathrm{AA} \mathrm{A}^{-1} \mathrm{Y}^{-1} \mathrm{~A}\right) \\
& =\lambda_{\mu}^{+}\left(\left(\mathrm{A}^{-1} \mathrm{X} \mathrm{A}\right)\left(\mathrm{A}^{-1} \mathrm{Y}^{-1} \mathrm{~A}\right)\right) \\
& =\lambda_{\mu}^{+}\left(\left(\mathrm{A}^{-1} \mathrm{XA}\right)\left(\mathrm{A}^{-1} \mathrm{Y}^{-1} \mathrm{~A}\right)^{-1}\right) \\
& \geq \min \left\{\lambda_{\mu}^{+}\left(\mathrm{A}^{-1} \mathrm{X} \mathrm{A}\right), \lambda_{\mu}^{+}\left(\mathrm{A}^{-1} \mathrm{Y}^{-1} \mathrm{~A}\right)^{-1}\right\} \\
& \geq \min \left\{\lambda_{\mu}^{+}\left(\mathrm{A}^{-1} \mathrm{XA}\right), \lambda_{\mu}^{+}\left(\mathrm{A}^{-1} \mathrm{Y}^{-1} \mathrm{~A}\right)\right\}, \\
& \quad\left(\text { since } \lambda_{\mu} \text { is a bipolar fuzzy HX subgroup of } \vartheta\right)
\end{aligned}
$$$$
\geq \min \left\{\left(\mathrm{A} \lambda_{\mu}{ }^{+} \mathrm{A}^{-1}\right)(\mathrm{X}),\left(\mathrm{A} \lambda_{\mu}{ }^{+} \mathrm{A}^{-1}\right)(\mathrm{Y})\right\} .
$$

Therefore, $\left(\mathrm{A} \lambda_{\mu}{ }^{+} \mathrm{A}^{-1}\right)\left(\mathrm{XY} \mathrm{Y}^{-1}\right) \geq \min \left\{\left(\mathrm{A} \lambda_{\mu}{ }^{+} \mathrm{A}^{-1}\right)(\mathrm{X}),\left(\mathrm{A} \lambda_{\mu}{ }^{+} \mathrm{A}^{-1}\right)(\mathrm{Y})\right\}$.

ii. $\left(A \lambda_{\mu}^{-} \mathrm{A}^{-1}\right)\left(\mathrm{XY}^{-1}\right)=\lambda_{\mu}^{-}\left(\mathrm{A}^{-1} \mathrm{XY} \mathrm{Y}^{-1} \mathrm{~A}\right)$, by the definition

$$
\begin{aligned}
& =\lambda_{\mu}^{-}\left(\mathrm{A}^{-1} \mathrm{XAA} \mathrm{A}^{-1} \mathrm{Y}^{-1} \mathrm{~A}\right) \\
& =\lambda_{\mu}{ }^{-}\left(\left(\mathrm{A}^{-1} \mathrm{XA}\right)\left(\mathrm{A}^{-1} \mathrm{Y}^{-1} \mathrm{~A}\right)\right) \\
& =\lambda_{\mu}^{-}\left(\left(\mathrm{A}^{-1} \mathrm{XA}\right)\left(\mathrm{A}^{-1} \mathrm{Y}^{-1} \mathrm{~A}\right)^{-1}\right) \\
& \leq \max \left\{\lambda_{\mu}^{-}\left(\mathrm{A}^{-1} \mathrm{XA}\right), \lambda_{\mu}^{-}\left(\mathrm{A}^{-1} \mathrm{Y}^{-1} \mathrm{~A}\right)^{-1}\right\} \\
& \leq \max \left\{\lambda_{\mu}^{-}\left(\mathrm{A}^{-1} \mathrm{XA}\right), \lambda_{\mu}^{-}\left(\mathrm{A}^{-1} \mathrm{Y}^{-1} \mathrm{~A}\right)\right\},
\end{aligned}
$$

(since $\lambda_{\mu}$ is a bipolar fuzzy subgroup of $\vartheta$ )

$$
\leq \max \left\{\left(\mathrm{A} \lambda_{\mu}^{-} \mathrm{A}^{-1}\right)(\mathrm{X}),\left(\mathrm{A} \lambda_{\mu}^{-} \mathrm{A}^{-1}\right)(\mathrm{Y})\right\}
$$

Therefore, $\left(\mathrm{A} \lambda_{\mu}^{-} \mathrm{A}^{-1}\right)\left(\mathrm{XY}^{-1}\right) \leq \max \left\{\left(\mathrm{A} \lambda_{\mu}^{-} \mathrm{A}^{-1}\right)(\mathrm{X}),\left(\mathrm{A} \lambda_{\mu}^{-} \mathrm{A}^{-1}\right)(\mathrm{Y})\right\}$.

Hence, $\left(A \lambda_{\mu} A^{-1}\right)=\left(A \lambda_{\mu}^{+} A^{-1}, A \lambda_{\mu}^{-} A^{-1}\right)$ is a bipolar fuzzy HX subgroup of $\vartheta$.

\subsection{Theorem}

Let $\mu=\left(\mu^{+}, \mu^{-}\right)$be a bipolar fuzzy subset on $\mathrm{G}$ and $\lambda_{\mu}=\left(\lambda_{\mu}{ }^{+}, \lambda_{\mu}{ }^{-}\right)$be a bipolar anti fuzzy HX subgroup of $\vartheta$ then the bipolar middle coset $\left(A \mu A^{-1}\right)=\left(A \mu^{+} A^{-1}, A \mu^{-} A^{-1}\right)$ is a bipolar anti fuzzy HX subgroup of $\vartheta$.

Proof : Let $\lambda_{\mu}=\left(\lambda_{\mu}{ }^{+}, \lambda_{\mu}{ }^{-}\right)$be a bipolar anti fuzzy HX subgroup of $\vartheta$ and Let for some $\mathrm{A} \in \vartheta$, for every $\mathrm{X}, \mathrm{Y} \in$ $\vartheta$ then

$$
\text { i. } \begin{aligned}
\left(\mathrm{A} \lambda_{\mu}{ }^{+} \mathrm{A}^{-1}\right)\left(\mathrm{XY}^{-1}\right) & =\lambda_{\mu}^{+}\left(\mathrm{A}^{-1} \mathrm{XY}^{-1} \mathrm{~A}\right), \text { by the definition } \\
& =\lambda_{\mu}^{+}\left(\mathrm{A}^{-1} \mathrm{XAA}^{-1} \mathrm{Y}^{-1} \mathrm{~A}\right) \\
& =\lambda_{\mu}^{+}\left(\left(\mathrm{A}^{-1} \mathrm{XA}\right)\left(\mathrm{A}^{-1} \mathrm{Y}^{-1} \mathrm{~A}\right)\right) \\
& =\lambda_{\mu}^{+}\left(\left(\mathrm{A}^{-1} \mathrm{XA}\right)\left(\mathrm{A}^{-1} \mathrm{Y}^{-1} \mathrm{~A}\right)^{-1}\right) \\
& \leq \max \left\{\lambda_{\mu}^{+}\left(\mathrm{A}^{-1} \mathrm{XA}\right), \lambda_{\mu}{ }^{+}\left(\mathrm{A}^{-1} \mathrm{Y}^{-1} \mathrm{~A}\right)^{-1}\right\} \\
& \leq \max \left\{\lambda_{\mu}^{+}\left(\mathrm{A}^{-1} \mathrm{XA}\right), \lambda_{\mu}^{+}\left(\mathrm{A}^{-1} \mathrm{Y}^{-1} \mathrm{~A}\right)\right\}, \\
& \quad\left(\text { since } \lambda_{\mu} \text { is a bipolar anti fuzzy subgroup of } \vartheta\right) \\
& \leq \max \left\{\left(\mathrm{A} \lambda_{\mu}^{+} \mathrm{A}^{-1}\right)(\mathrm{X}),\left(\mathrm{A} \lambda_{\mu}^{+} \mathrm{A}^{-1}\right)(\mathrm{Y})\right\} .
\end{aligned}
$$

Therefore, $\left(\mathrm{A} \lambda_{\mu}{ }^{+} \mathrm{A}^{-1}\right)\left(\mathrm{XY}^{-1}\right) \leq \max \left\{\left(\mathrm{A} \lambda_{\mu}^{+} \mathrm{A}^{-1}\right)(\mathrm{X}),\left(\mathrm{A} \lambda_{\mu}{ }^{+} \mathrm{A}^{-1}\right)(\mathrm{Y})\right\}$.

$$
\text { ii. } \begin{aligned}
\left(\mathrm{A} \lambda_{\mu}{ }^{-} \mathrm{A}^{-1}\right)\left(\mathrm{XY}^{-1}\right) & =\lambda_{\mu}^{-}\left(\mathrm{A}^{-1} \mathrm{XY} \mathrm{Y}^{-1} \mathrm{~A}\right), \text { by the definition } \\
& =\lambda_{\mu}^{-}\left(\mathrm{A}^{-1} \mathrm{XAA}^{-1} \mathrm{Y}^{-1} \mathrm{~A}\right) \\
& =\lambda_{\mu}^{-}\left(\left(\mathrm{A}^{-1} \mathrm{XA}\right)\left(\mathrm{A}^{-1} \mathrm{Y}^{-1} \mathrm{~A}\right)\right) \\
& =\lambda_{\mu}^{-}\left(\left(\mathrm{A}^{-1} \mathrm{XA}\right)\left(\mathrm{A}^{-1} \mathrm{Y}^{-1} \mathrm{~A}\right)^{-1}\right) \\
& \geq \min \left\{\lambda_{\mu}^{-}\left(\mathrm{A}^{-1} \mathrm{XA}\right), \lambda_{\mu}{ }^{-}\left(\mathrm{A}^{-1} \mathrm{Y}^{-1} \mathrm{~A}\right)^{-1}\right\} \\
& \geq \min \left\{\lambda_{\mu}^{-}\left(\mathrm{A}^{-1} \mathrm{XA}\right), \lambda_{\mu}^{-}\left(\mathrm{A}^{-1} \mathrm{Y}^{-1} \mathrm{~A}\right)\right\}, \\
& \quad\left(\text { since } \lambda_{\mu} \text { is a bipolar anti fuzzy HX subgroup of } \vartheta\right) \\
& \geq \min \left\{\left(\mathrm{A} \lambda_{\mu}^{-} \mathrm{A}^{-1}\right)(\mathrm{X}),\left(\mathrm{A} \lambda_{\mu}^{-} \mathrm{A}^{-1}\right)(\mathrm{Y})\right\} .
\end{aligned}
$$

Therefore, $\left(A \lambda_{\mu}{ }^{-} \mathrm{A}^{-1}\right)\left(X \mathrm{XY}^{-1}\right) \geq \min \left\{\left(\mathrm{A} \lambda_{\mu}{ }^{-} \mathrm{A}^{-1}\right)(\mathrm{X}),\left(\mathrm{A} \lambda_{\mu}{ }^{-} \mathrm{A}^{-1}\right)(\mathrm{Y})\right\}$.

Hence, $\left(A \lambda_{\mu} A^{-1}\right)=\left(A \lambda_{\mu}^{+} A^{-1}, A \lambda_{\mu}^{-} A^{-1}\right)$ is a bipolar anti fuzzy HX subgroup of $\vartheta$. 


\subsection{Definition}

Let $\mu=\left(\mu^{+}, \mu^{-}\right)$and $\varphi=\left(\varphi^{+}, \varphi^{-}\right)$are two bipolar fuzzy subsets of a group $G$ and $\lambda_{\mu}=\left(\lambda_{\mu}{ }^{+}, \lambda_{\mu}{ }^{-}\right)$, $\lambda_{\varphi}=\left(\lambda_{\varphi}{ }^{+}, \lambda_{\varphi}{ }^{-}\right)$are two bipolar fuzzy HX subgroups (bipolar anti fuzzy HX subgroups) of $\vartheta$. Then $\lambda_{\mu}$ and $\lambda_{\varphi}$ are said to be conjugate bipolar fuzzy HX subgroups (conjugate bipolar anti fuzzy HX subgroups) of $\vartheta$ if for some $\mathrm{A} \in \vartheta$,

$$
\begin{array}{ll}
\text { i. } & \lambda_{\mu}{ }^{+}(\mathrm{X})=\lambda_{\varphi}{ }^{+}\left(\mathrm{A}^{-1} \mathrm{XA}\right) \\
\text { ii. } & \lambda_{\mu}{ }^{-}(\mathrm{X})=\lambda_{\varphi}{ }^{-}\left(\mathrm{A}^{-1} \mathrm{XA}\right), \text { for every } \mathrm{X} \in \vartheta .
\end{array}
$$

\subsection{Theorem}

Let $\lambda_{\mu}=\left(\lambda_{\mu}{ }^{+}, \lambda_{\mu}{ }^{-}\right)$and $\lambda_{\varphi}=\left(\lambda_{\varphi}{ }^{+}, \lambda_{\varphi}{ }^{-}\right)$are two bipolar fuzzy HX subgroups of an abelian HX group $\vartheta$ if and only if $\lambda_{\mu}=\lambda_{\varphi}$.

Proof : Let $\lambda_{\mu}=\left(\lambda_{\mu}{ }^{+}, \lambda_{\mu}{ }^{-}\right)$and $\lambda_{\varphi}=\left(\lambda_{\varphi}{ }^{+}, \lambda_{\varphi}{ }^{-}\right)$be any two conjugate bipolar fuzzy HX subgroups of an abelian HX group $\vartheta$, then for some $B \in \vartheta$,

$\lambda_{\mu}{ }^{+}(\mathrm{A})=\lambda_{\varphi}{ }^{+}\left(\mathrm{B}^{-1} \mathrm{AB}\right)$, for every $\mathrm{A} \in \vartheta$

$\Leftrightarrow \quad \lambda_{\mu}^{+}(\mathrm{A}) \quad=\lambda_{\varphi}{ }^{+}\left(\mathrm{B}^{-1} \mathrm{BA}\right)$, since $\vartheta$ is abelian

$\Leftrightarrow \lambda_{\mu}^{+}(\mathrm{A}) \quad=\lambda_{\varphi}^{+}(\mathrm{EA})$

$\Leftrightarrow \quad \lambda_{\mu}^{+}(\mathrm{A}) \quad=\lambda_{\varphi}{ }^{+}(\mathrm{A})$

ii. $\quad \lambda_{\mu}{ }^{-}(\mathrm{A})=\lambda_{\varphi}{ }^{-}\left(\mathrm{B}^{-1} \mathrm{AB}\right)$, for every $\mathrm{A} \in \vartheta$

$\Leftrightarrow \quad \lambda_{\mu}{ }^{-}(\mathrm{A}) \quad=\lambda_{\varphi}{ }^{-}\left(\mathrm{B}^{-1} \mathrm{BA}\right)$, since $\vartheta$ is abelian

$\Leftrightarrow \lambda_{\mu}^{-}(\mathrm{A}) \quad=\lambda_{\varphi}{ }^{-}(\mathrm{EA})$

$\Leftrightarrow \quad \lambda_{\mu}{ }^{-}(\mathrm{A}) \quad=\lambda_{\varphi}{ }^{-}(\mathrm{A})$

Hence, $\lambda_{\mu}=\lambda_{\varphi} \Leftrightarrow \lambda_{\mu}=\left(\lambda_{\mu}{ }^{+}, \lambda_{\mu}{ }^{-}\right)$and $\lambda_{\varphi}=\left(\lambda_{\varphi}{ }^{+}, \lambda_{\varphi}{ }^{-}\right)$are conjugate bipolar fuzzy HX subgroups of an abelian group $\vartheta$.

\subsection{Theorem}

Let $\lambda_{\mu}=\left(\lambda_{\mu}{ }^{+}, \lambda_{\mu}{ }^{-}\right)$and $\lambda_{\varphi}=\left(\lambda_{\varphi}{ }^{+}, \lambda_{\varphi}{ }^{-}\right)$are two bipolar anti fuzzy HX subgroups of an abelian HX group $\vartheta$ if and only if $\lambda_{\mu}=\lambda_{\varphi}$.

Proof : Similar to Theorem 2.20.

\section{PROPERTIES OF LEVEL SUBSETS AND LOWER LEVEL SUBSETS OF A BIPOLAR FUZZY HX SUBGROUP AND BIPOLAR ANTI FUZZY HX SUBGROUP}

In this section, we introduce the concept of level subsets of a bipolar fuzzy cosets of bipolar fuzzy HX subgroup and lower level subsets of a bipolar fuzzy cosets of a bipolar anti fuzzy HX subgroup and discuss some of its properties.

\subsection{Definition}

Let $\mu=\left(\mu^{+}, \mu^{-}\right)$be a bipolar fuzzy subset of G. For any $<\alpha, \beta>\in[0,1] \times[-1,0]$, the bipolar set $\mathrm{U}\left[\mu_{<\alpha, \beta>}\right]=\left\{\mathrm{x} \in \mathrm{G} / \mu^{+}(\mathrm{x}) \geq \alpha\right.$ and $\left.\mu^{-}(\mathrm{x}) \leq \beta\right\}$ is called a level subset of the bipolar fuzzy subset $\mu$.

\subsection{Definition [7]}

Let $\lambda_{\mu}=\left(\lambda_{\mu}{ }^{+}, \lambda_{\mu}{ }^{-}\right)$be a bipolar fuzzy HX subgroup of a HX group $\vartheta$. For any $<\alpha, \beta>\in[0,1] \times[-1,0]$, we define the set $\mathrm{U}\left[\lambda_{\mu<\alpha, \beta>}\right]=\left\{\mathrm{A} \in \vartheta / \lambda_{\mu}{ }^{+}(\mathrm{A}) \geq \alpha\right.$ and $\left.\lambda_{\mu}{ }^{-}(\mathrm{A}) \leq \beta\right\}$ is called the $<\alpha, \beta>$ level subset of $\lambda_{\mu}$ or simply the level subset of $\lambda_{\mu}$.

\subsection{Definition}

Let $\lambda_{\mu}=\left(\lambda_{\mu}{ }^{+}, \lambda_{\mu}{ }^{-}\right)$be a bipolar fuzzy HX subgroup of a HX group $\vartheta$.For any $<\alpha, \beta>\in[0,1] \times[-1,0]$, we define the set $\mathrm{U}\left[\mathrm{A} \lambda_{\mu<\alpha, \beta}\right]=\left\{\mathrm{X} \in \vartheta /\left(\mathrm{A} \lambda_{\mu}{ }^{+}\right)(\mathrm{X})=\lambda_{\mu}{ }^{+}\left(\mathrm{A}^{-1} \mathrm{X}\right) \geq \alpha\right.$ and $\left(\mathrm{A} \lambda_{\mu}{ }^{-}\right)(\mathrm{X})=\lambda_{\mu}{ }^{-}\left(\mathrm{A}^{-1} \mathrm{X}\right) \leq \beta$, for some $A \in \vartheta$ \} is called the level subset of a bipolar fuzzy coset $\left(A \lambda_{\mu}\right)$.

\subsection{Definition}

Let $\mu=\left(\mu^{+}, \mu^{-}\right)$be a bipolar fuzzy subset of G. For any $<\alpha, \beta>\in[0,1] \times[-1,0]$, the bipolar set $\mathrm{L}\left[\mu_{<\alpha, \beta}\right]=\left\{\mathrm{x} \in \mathrm{G} / \mu^{+}(\mathrm{x}) \leq \alpha\right.$ and $\left.\mu^{-}(\mathrm{x}) \geq \beta\right\}$ is called a lower level subset of the bipolar fuzzy subset $\mu$.

\subsection{Definition [6]}

Let $\lambda_{\mu}=\left(\lambda_{\mu}{ }^{+}, \lambda_{\mu}{ }^{-}\right)$be a bipolar anti fuzzy HX subgroup of a HX group $\vartheta$. For any $\left\langle\alpha, \beta>\in[0,1] \times[-1,0]\right.$, we define the set $L\left[\lambda_{\mu<\alpha, \beta}\right]=\left\{A \in \vartheta / \lambda_{\mu}{ }^{+}(\mathrm{A}) \leq \alpha\right.$ and $\left.\lambda_{\mu}{ }^{-}(\mathrm{A}) \geq \beta\right\}$ is called the $<\alpha, \beta>$ lower level subset of $\lambda_{\mu}$ or simply the lower level subset of $\lambda_{\mu}$. 


\subsection{Definition}

Let $\lambda_{\mu}=\left(\lambda_{\mu}{ }^{+}, \lambda_{\mu}{ }^{-}\right)$be a bipolar anti fuzzy HX subgroup of a HX group $\vartheta$. For any $<\alpha, \beta>\in[0,1] \times[-1,0]$, we define the set $\mathrm{L}\left[\mathrm{A} \lambda_{\mu<\alpha, \beta>}\right]=\left\{\mathrm{X} \in \vartheta /\left(\mathrm{A} \lambda_{\mu}{ }^{+}\right)(\mathrm{X})=\lambda_{\mu}{ }^{+}\left(\mathrm{A}^{-1} \mathrm{X}\right) \leq \alpha\right.$ and $\left(\mathrm{A} \lambda_{\mu}{ }^{-}\right)(\mathrm{X})=$ $\lambda_{\mu}{ }^{-}\left(A^{-1} X\right) \geq \beta$, for some $\left.A \in \vartheta\right\}$ is called the lower level subset of a bipolar fuzzy coset $\left(A \lambda_{\mu}\right)$.

\subsection{Theorem}

Let $\mu=\left(\mu^{+}, \mu^{-}\right)$be a bipolar fuzzy subset on $\mathrm{G}$ and $\lambda_{\mu}=\left(\lambda_{\mu}{ }^{+}, \lambda_{\mu}{ }^{-}\right)$be a bipolar fuzzy HX subgroup of $\vartheta$ then $\mathrm{U}\left[\mathrm{A} \lambda_{\mu<\alpha, \beta}\right]=\mathrm{AU}\left[\lambda_{\mu<\alpha, \beta>}\right]$ for every $\mathrm{A} \in \vartheta$ and $\langle\alpha, \beta>\in[0,1] \times[-1,0]$.

Proof : Let $\lambda_{\mu}=\left(\lambda_{\mu}{ }^{+}, \lambda_{\mu}{ }^{-}\right)$be a bipolar fuzzy HX subgroup of $\vartheta$ and Let $X \in \vartheta$

Now $\mathrm{U}\left[\mathrm{A} \lambda_{\mu<\alpha, \beta}\right]=\mathrm{U}\left[\left(\mathrm{A} \lambda_{\mu}{ }^{+} ; \alpha\right),\left(\mathrm{A} \lambda_{\mu}{ }^{-} ; \beta\right)\right]$

Let $\mathrm{X} \in \mathrm{U}\left[\mathrm{A} \lambda_{\mu<\alpha, \beta>}\right] \Rightarrow \mathrm{X} \in \mathrm{U}\left(\mathrm{A} \lambda_{\mu}{ }^{+} ; \alpha\right)$ and $\mathrm{X} \in \mathrm{U}\left(\mathrm{A} \lambda_{\mu}{ }^{-} ; \beta\right)$

i. $\quad \mathrm{X} \in \mathrm{U}\left(\mathrm{A} \lambda_{\mu}^{+} ; \alpha\right) \Leftrightarrow\left(\mathrm{A} \lambda_{\mu}^{+}\right)(\mathrm{X}) \geq \alpha$

$$
\begin{aligned}
& \Leftrightarrow \lambda_{\mu}^{+}\left(\mathrm{A}^{-1} \mathrm{X}\right) \geq \alpha \\
& \Leftrightarrow \mathrm{A}^{-1} \mathrm{X} \in \mathrm{U}\left(\lambda_{\mu}^{+} ; \alpha\right) \\
& \Leftrightarrow \mathrm{X} \in \mathrm{AU}\left(\lambda_{\mu}^{+} ; \alpha\right)
\end{aligned}
$$

Therefore, $\mathrm{U}\left(\mathrm{A} \lambda_{\mu}{ }^{+} ; \alpha\right)=\mathrm{A} \mathrm{U}\left(\lambda_{\mu}{ }^{+} ; \alpha\right)$, for every $\mathrm{A} \in \vartheta$

$$
\text { ii. } \quad \begin{aligned}
\mathrm{X} \in \mathrm{U}\left(\mathrm{A} \lambda_{\mu}^{-} ; \beta\right) & \Leftrightarrow\left(\mathrm{A} \lambda_{\mu}^{-}\right)(\mathrm{X}) \leq \beta \\
& \Leftrightarrow \lambda_{\mu}^{-}\left(\mathrm{A}^{-1} \mathrm{X}\right) \leq \beta \\
& \Leftrightarrow \mathrm{A}^{-1} \mathrm{X} \in \mathrm{U}\left(\lambda_{\mu}^{-} ; \beta\right) \\
& \Leftrightarrow \mathrm{X} \in \mathrm{AU}\left(\lambda_{\mu}^{-} ; \beta\right)
\end{aligned}
$$

Therefore, $\mathrm{U}\left(\mathrm{A} \lambda_{\mu}{ }^{-} ; \beta\right)=\mathrm{A} \mathrm{U}\left(\lambda_{\mu}{ }^{-} ; \beta\right)$, for every $\mathrm{A} \in \vartheta$.

From these, $\quad \mathrm{U}\left[\mathrm{A} \lambda_{\mu<\alpha, \beta>}\right]=\mathrm{A} \mathrm{U}\left[\lambda_{\mu<\alpha, \beta}\right]$

\subsection{Theorem}

Let $\mu=\left(\mu^{+}, \mu^{-}\right)$be a bipolar fuzzy subset on $\mathrm{G}$ and $\lambda_{\mu}=\left(\lambda_{\mu}{ }^{+}, \lambda_{\mu}{ }^{-}\right)$be a bipolar anti fuzzy HX subgroup of $\vartheta$ then $\mathrm{L}\left[\mathrm{A} \lambda_{\mu<\alpha, \beta}\right]=\mathrm{AL}\left[\lambda_{\mu<\alpha, \beta\rangle}\right]$ for every $\mathrm{A} \in \vartheta$ and $\langle\alpha, \beta>\in[0,1] \times[-1,0]$.

Proof : Let $\lambda_{\mu}=\left(\lambda_{\mu}{ }^{+}, \lambda_{\mu}{ }^{-}\right)$be a bipolar fuzzy HX subgroup of $\vartheta$ and Let $X \in \vartheta$

Now $\mathrm{L}\left[\mathrm{A} \lambda_{\mu<\alpha, \beta>}\right]=\mathrm{L}\left[\left(\mathrm{A} \lambda_{\mu}{ }^{+} ; \alpha\right),\left(\mathrm{A} \lambda_{\mu}{ }^{-} ; \beta\right)\right]$

Let $\mathrm{X} \in \mathrm{L}\left[\mathrm{A} \lambda_{\mu<\alpha, \beta>}\right] \Rightarrow \mathrm{X} \in \mathrm{L}\left(\mathrm{A} \lambda_{\mu}^{+} ; \alpha\right)$ and $\mathrm{X} \in \mathrm{L}\left(\mathrm{A} \lambda_{\mu}{ }^{-} ; \beta\right)$

i. $\quad \mathrm{X} \in \mathrm{L}\left(\mathrm{A} \lambda_{\mu}^{+} ; \alpha\right) \Leftrightarrow\left(\mathrm{A} \lambda_{\mu}^{+}\right)(\mathrm{X}) \leq \alpha$

$\Leftrightarrow \lambda_{\mu}^{+}\left(\mathrm{A}^{-1} \mathrm{X}\right) \leq \alpha$

$\Leftrightarrow \mathrm{A}^{-1} \mathrm{X} \in \mathrm{L}\left(\lambda_{\mu}^{+} ; \alpha\right)$

$\Leftrightarrow \mathrm{X} \in \mathrm{AL}\left(\lambda_{\mu}^{+} ; \alpha\right)$

Therefore, $\mathrm{L}\left(\mathrm{A} \lambda_{\mu}{ }^{+} ; \alpha\right)=\mathrm{AL}\left({\lambda_{\mu}}^{+} ; \alpha\right)$, for every $\mathrm{A} \in \vartheta$

ii. $\quad \mathrm{X} \in \mathrm{L}\left(\mathrm{A} \lambda_{\mu}^{-} ; \beta\right) \Leftrightarrow\left(\mathrm{A} \lambda_{\mu}^{-}\right)(\mathrm{X}) \geq \beta$

$\Leftrightarrow \lambda_{\mu}^{-}\left(\mathrm{A}^{-1} \mathrm{X}\right) \geq \beta$

$\Leftrightarrow \mathrm{A}^{-1} \mathrm{X} \in \mathrm{L}\left(\lambda_{\mu}^{-} ; \beta\right)$

$\Leftrightarrow X \in \operatorname{AL}\left(\lambda_{\mu}^{-} ; \beta\right)$

Therefore, $\mathrm{L}\left(\mathrm{A} \lambda_{\mu}{ }^{-} ; \beta\right)=\operatorname{AL}\left(\lambda_{\mu}{ }^{-} ; \beta\right)$, for every $\mathrm{A} \in \vartheta$.

From these, $\quad \mathrm{L}\left[\mathrm{A} \lambda_{\mu<\alpha, \beta>}\right]=\mathrm{A}\left[\lambda_{\mu<\alpha, \beta>}\right]$

\section{IV.}

CONCLUSION

In this paper, we introduced the notion of bipolar fuzzy cosets of a bipolar fuzzy and bipolar anti fuzzy HX subgroups of a HX group with suitable examples. We extended these ideas to the bipolar fuzzy quotient HX subgroup and discussed few important properties of them.

\section{Acknowledgement}

Authors wish to thank all our friends for their help to make this paper as a successful one. 


\section{REFERENCES}

\section{Journal Papers:}

[1]. R. Biswas, Fuzzy subgroups and anti-fuzzy subgroups, Fuzzy Sets and Systems , 35(1990), 121-124.

[2]. V.N. Dixit ,A. Rajesh Kumar, Naseem Ajmal, Level subgroups and union of Fuzzy Subgroups, Fuzzy Sets and Systems, 37,359 371 (1990).

[3]. Li Hongxing, HX group, BUSEFAL, 33 October 1987, 31 - 37.

[4]. Luo Chengzhong, Mi Honghai , Li Hongxing, Fuzzy HX group, BUSEFAL , 41 _ 14, Oct 1989. 97 - 106.

[5]. M. Marudai, V. Rajendran, New Constructions on Bipolar Anti Q-fuzzy Groups and Bipolar Anti Q-fuzzy d-ideals under (t,s) norms, Advances in Fuzzy Mathematics, Volume 6, Number 1 (2011), pp .145 - 153.

[6]. R. Muthuraj, M. Sridharan, Bipolar Anti fuzzy HX group and its Lower Level Sub HX groups, Journal of Physical Sciences, Volume 16, December $2012,157-169$.

[7]. R. Muthuraj, M. Sridharan, Bipolar fuzzy HX group and its Level Sub HX groups, International Journal of Mathematical Archive, (Communicated).

[8]. L. A. Zadeh., Fuzzy sets, Information and control, 8, (1965) 338-353.

[9]. Prahibir Bhattacharya, Fuzzy sub groups: Some characterizations, J. Math. Anal. Appl. 128 (1981) 241-252.

[10]. A. Rosenfeld, Fuzzy Groups, Journal of Mathematical Analysis and Applications, 35(1971), 512-51.

\section{Books:}

[11]. W.B. Vasantha Kandasamy, Smarandahe Fuzzy Algebra, (American Research Press, 2003).

\section{Proceedings Papers:}

[12]. W.R. Zhang, Bipolar fuzzy sets, Proc. of FUZZ-IEEE, pp: 835-840, 1998. 